\title{
5'位羟基异戊烯基查尔酮类天然产物的合成及其抗菌活性研究
}

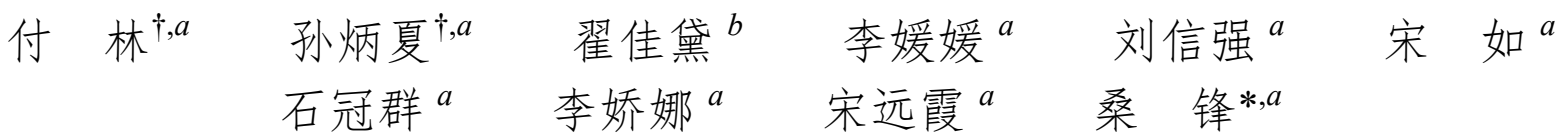 \\ $\left({ }^{a}\right.$ 山东理工大学生命科学学院 山东淄博 255049) \\ ( ${ }^{b}$ 天津大学药物科学与技术学院 天津 300072$)$
}

\begin{abstract}
摘要 首次合成了 Bartericin A (1), 2',6'-二羟基-5'-(2"-羟基-3"-甲基-3"-丁烯基)-4'-甲氧基查尔酮(2), Xanthohumol D (3) 和 Angusticornin B (4) 4 个羟基异戊烯基查尔酮类天然产物. 为了探讨天然产物中不同官能团对其核心骨架结构抗菌活 性的影响, 设计合成了衍生物 6. 所合成的目标产物和未知中间体化合物经过 ${ }^{1} \mathrm{H} N M R 、{ }^{13} \mathrm{C} N M R 、 I R 、 H R M S$ 进行了 确证. 选取大肠杆菌[CMCC(B)44102]、绿脓杆菌[CMCC(B) 10104]、金黄色葡萄球菌[CMCC(B)260003]和枯草芽孢杆菌 [CMCC(B)63 501], 采用稀释点样法对所合成的 4 个天然产物及 1 个新型衍生物进行了抗菌活性评估. 结果显示, 天然 产物 $1 、 4$ 和衍生物 6 对革兰氏阳性菌金黄色葡萄球菌和枯草芽狍杆菌表现出了一定的抑制活性. 天然产物 3 对枯草芽 狍杆菌表现出了较为明显的抑制活性，但对其他 3 种菌株无抑制活性(最小抑菌浓度 $>200 \mu \mathrm{g} / \mathrm{mL}$ ).
\end{abstract}

关键词＼cjkstart羟基异戊烯基; 查尔酮; 天然产物; 抗菌活性

\section{Synthesis and Antibacterial Activity Study of Natural 5'-Hydroxyisoprenyl Chalcones}

\author{
Fu, Lin ${ }^{\dagger, a} \quad$ Sun, Bingxia ${ }^{\dagger, a} \quad$ Zhai, Jiadai $^{b} \quad$ Li, Yuanyuan $^{a} \quad$ Liu, Xinqiang ${ }^{a}$ \\ Song, $\mathrm{Ru}^{a} \quad$ Shi, Guanqun $^{a} \quad \mathrm{Li}$, Jiaona ${ }^{a} \quad$ Song, Yuanxia ${ }^{a} \quad$ Sang, Feng ${ }^{*, a}$ \\ $\left({ }^{a}\right.$ School of Life Science, Shandong University of Technology, Zibo, Shangdong 255049) \\ $\left({ }^{b}\right.$ School of Pharmaceutical Science and Technology, Tianjin University, Tianjin 300072)
}

\begin{abstract}
Four natural chalcones, bartericin A (1), 2',6'-dihydroxy-5'-(2"-hydroxy-3"-methyl-3"-butenyl)-4'-methoxychalcone (2), xanthohumol D (3) and angusticornin B (4) were synthesized for the first time and all of them shared 5'-hydroxyisoprenyl group in common. One of their deriative, compound 6, was also prepared in order to investigate the effect of different functional group in natural products on antibacterial activity of the core structure. After confirming their structures by ${ }^{1} \mathrm{H}$ NMR, ${ }^{13} \mathrm{C}$ NMR, IR and HRMS, $1 \sim \mathbf{4}$ and $\mathbf{6}$ were evaluated for their antibacterial activities against Bacillus subtilis [CMCC(B)63 501], Staphylococcus aureus [CMCC(B)260003], Escherichia coli [CMCC(B)44102] and Pseudomonas aeruginosa [CMCC(B)10104]. In this assay micro-dilution method was employed. The results showed that compounds $\mathbf{1}, \mathbf{4}$ and 6 exhibited moderate activity against gram-positive bacteria Bacillus subtilis and Staphylococcus aureus. Meanwhile compound 3 showed significant activity towards Bacillus subtilis but no activity to the other 3 strains even in $200 \mu \mathrm{g} / \mathrm{mL}$ concentration.
\end{abstract}

Keywords hydroxyisoprenyl; chalcone; natural product; antibacterial activity

查尔酮类天然产物广泛存在于多种药用植物中, 具 有特殊的 1,3-二芳基丙烯酮骨架结构 ${ }^{[1]}$. 因其具有多种 重要的生物活性 ${ }^{[2 \sim 9]}$, 包括抗菌、抗肿瘤、杀虫、消炎
以及治疗糖尿病等, 在过去的几十年中, 新型天然产物 的提取分离和对其进行结构修饰研究从未间 ${ }^{-}{ }^{[10,11]}$. 2016 年, Shaffer 等从美国德克萨斯原生植物中提取分离

\footnotetext{
* Corresponding author. E-mail: fengsang@sdut.edu.cn

Received July 19, 2019; revised September 1, 2019; published online September 25, 2019.

Project supported by the Natural Science Foundation of Shandong Province (No. ZR2016HQ04), the National Natural Science Foundation of China (No. 81602962) and the China Postdoctoral Science Foundation (No. 2019M652461)

山东省自然科学基金(No. ZR2016HQ04)、国家自然科学基金(No. 81602962)和中国博士后科学基金(No. 2019M652461)资助项目.

$\dagger$ 共同第一作者(The authors contributed equally to this work).
} 
到一个新型羟基异戊烯基查尔酮类天然产物 Sanjuanolide (5) ${ }^{[12]}$, 并发现其具有较为显著的生物活性. 在我们前期的工作中, 研究了 $\mathbf{5}$ 及其衍生物的合成, 并 初步探讨了其抗肿瘤构效关系 ${ }^{[13]}$. 刘志国等 ${ }^{[14]}$ 完成了 5 的两个对映异构体的手性合成，并研究了其抗炎活性.

天然产物 5 及其衍生物中的羟基异戊烯基位于查尔 酮的 $3^{\prime}$ 位(如图 1 所示). 经数据库搜索后不难发现, 5'位 含有羟基异戊烯基的查尔酮类天然产物主要包括 Bartericin A (1) ${ }^{[15]}, 2^{\prime}, 6^{\prime}$-二羟基-5'-(2"-羟基-3"-甲基-3"-丁 烯基)-4'-甲氧基查尔酮(2) ${ }^{[16]}$, Xanthohumol D (3) ${ }^{[17]}$ 和 Angusticornin B (4) ${ }^{[18]}$, 其化学合成工作目前尚未见报 道. 前期初步研究发现, 天然产物 Sanjuanolide (5)具有 较为显著的抑菌活性, 因此合成 4 个 5 位含有羟基异成 烯基的查尔酮类天然产物, 并探究其抗菌活性, 具有较 为重要的研究意义.
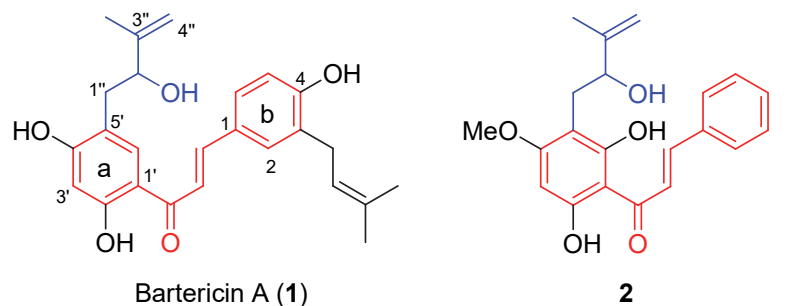<smiles>C=C(C)C(O)Cc1c(O)cc(OC)c(C(=O)/C=C/c2ccc(O)cc2)c1O</smiles>

Xanthohumol D (3)<smiles>C=C(C)C(O)Cc1c(O)ccc(C(=O)/C=C/c2ccccc2)c1O</smiles>

Sanjuanolide (5)
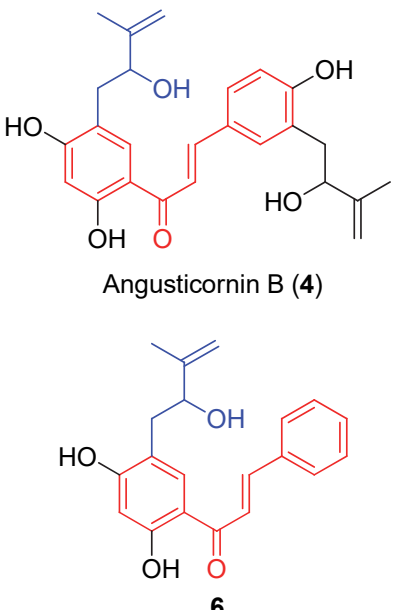

图 1 查尔酮类天然产物及其衍生物 $1 \sim 6$ 的结构

Figure 1 Structures of natural chalcone and their derivatives $1 \sim 6$

\section{1 结果与讨论}

\section{1 目标化合物的合成}

天然产物 1 的合成由已知化合物 $7^{[19]}$ 作为起始原料, 在可见光的催化下发生 Schenckene 反应, 得到化合物 $\mathbf{8}$, 8 再与已知化合物 $\mathbf{9}^{[20]}$ 在碱性条件下发生 ClaisenSchmidt 缩合反应, 得到化合物 10. 化合物 10 在对甲苯 磺酸的作用下加热脱去两个保护基得到目标产物 $\mathbf{1}$.

天然产物 $\mathbf{2}$ 的合成以化合物 $\mathbf{1 1}^{[21]}$ 为起始原料. 首先
引入甲氧基甲基(MOM)保护得到化合物 12，12 再与苯 甲醛发生 Claisen-Schmidt缩合反应得到化合物 13. 化合 物 13 再经过 Schenckene 反应得到化合物 14，最后脱去 保护得到目标产物 2.<smiles>C=C(C)C(O)Cc1cc(C(C)=O)c(O)cc1OCC(=O)OCC(C)C</smiles><smiles>C=C(C)C(O)Cc1cc(C(=O)/C=C/c2ccc(OC)c(CC=C(C)C)c2)c(O)cc1OC</smiles>

$\underset{50-T S A, ~ T H F-M e O H}{ }$<smiles>C=C(C)C(O)Cc1cc(C(=O)C=Cc2ccc(O)c(CC=C(C)C)c2)c(O)cc1O</smiles>

图式 1 天然产物 1 的合成路线

Scheme 1 Synthetic route of natural product 1

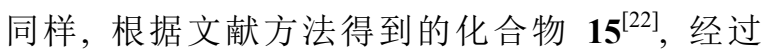
Schenckene 和脱保护两步反应得到天然产物 3. 天然产 物 4 可以由已知天然产物 Stipulin (17 $)^{[23]}$ 直接通过 Schenckene 反应制得.

以化合物 7 作为起始原料，与苯甲醛发生 ClaisenSchmidt 缩合反应得到化合物 18, 18 先脱去 MOM 保护 基，再经过 Schenck ene 反应后得到目标化合物 $\mathbf{6}$.

\section{2 抗菌活性测试}

以氨苠西林作为阳性对照, 以大肠杆菌 $[\mathrm{CMCC}(\mathrm{B})-$ 44102]、绿脓杆菌 $[\mathrm{CMCC}(\mathrm{B}) 10104]$ 、金黄色葡萄球菌 [CMCC(B)260003]和枯草芽孢杆菌[CMCC(B)63 501]为 待测菌株, 采用稀释点样法测定目标化合物对待测菌株 的抑制活性，结果见表 1. 测试结果表明，化合物 1、4、 6 对革兰氏阳性菌金黄色葡萄球菌和枯草芽孢杆菌表现 出一定的抑制活性 $(25 \sim 75 \mu \mathrm{g} / \mathrm{mL})$. 天然产物 3 对枯草 芽孢杆菌表现出较为明显的选择性抑制活性(12.5 $\mu \mathrm{g} / \mathrm{mL}$ ), 而对其他 3 种菌株无抑制活性 (MIC $>200$ $\mu \mathrm{g} / \mathrm{mL})$.

由表 1 中的数据分析可知, 查尔酮 3 位上异戊烯基 被氧化为羟基异戊烯基后，抗菌活性略有下降(1 和 4 相 
表 1 天然产物 $\mathbf{1} \sim \mathbf{4}$ 和衍生物 $\mathbf{6}$ 的抗菌活性(最小抑菌浓度, $\mu \mathrm{g} / \mathrm{mL}$ )

Table 1 Inhibitory activity (MIC, $\mu \mathrm{g} / \mathrm{mL}$ ) of natural products $\mathbf{1} \sim \mathbf{4}$ and derivative $\mathbf{6}$ against bacteria

\begin{tabular}{ccccc}
\hline Compd. & E. coli 44102 & P. aeruginosa 10104 & S. aureus 260003 & B. subtilis 63 501 \\
\hline $\mathbf{1}$ & $>200$ & $>200$ & 25 & 25 \\
$\mathbf{2}$ & $>200$ & $>200$ & $>200$ & $>200$ \\
$\mathbf{3}$ & $>200$ & $>200$ & $>200$ & 12.5 \\
$\mathbf{4}$ & $>200$ & $>200$ & 50 & 50 \\
$\mathbf{6}$ & $>200$ & $>200$ & 75 & 75 \\
Ampicillin & 62.5 & 62.5 & 3.125 & 6.25 \\
\hline
\end{tabular}<smiles>COc1cc(O)c(C(C)=O)c(O)c1CC=C(C)C</smiles><smiles>COc1cc(OC)c(C(C)=O)c(O)c1CC=C(C)C</smiles>

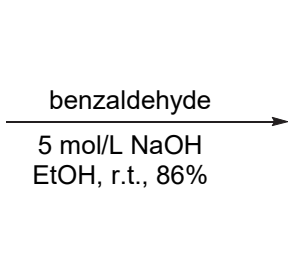<smiles>COc1cc(OC)c(C(=O)C=Cc2ccccc2)c(O)c1CC=C(C)C</smiles>

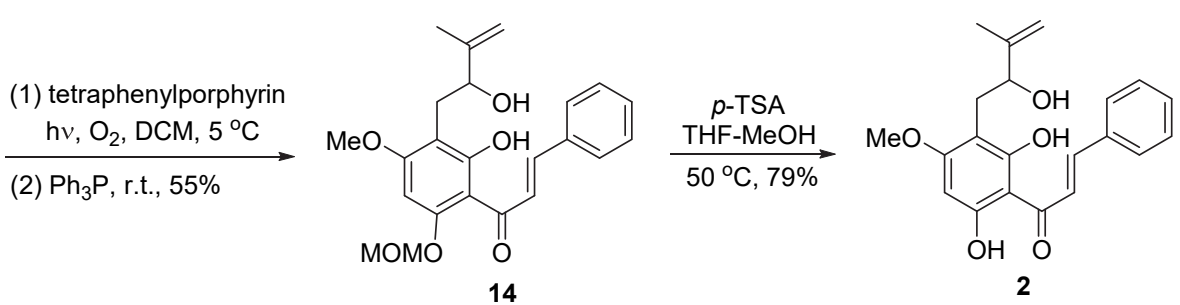

图式 2 天然产物 2 的合成路线

Scheme 2 Synthetic route of natural product 2<smiles>COc1ccc(/C=C/C(=O)c2c(OC)cc(OC)c(CC=C(C)C)c2OC)cc1</smiles>

15
(1) tetraphenylporphyrin $\mathrm{hv}, \mathrm{O}_{2}$, DCM, $5^{\circ} \mathrm{C}$

(2) $\mathrm{Ph}_{3}$ P, r.t., $42 \%$ MOMO<smiles>C=C(C)C(O)Cc1c(OC)cc(OC)c(C(=O)/C=C/c2ccc(OC)cc2)c1OC</smiles>

16 $\underset{50^{\circ} \mathrm{C}, 76 \%}{\stackrel{p-T S A}{\longrightarrow} \text { THF-MeOH }}$<smiles>C=C(C)C(O)Cc1c(O)cc(OC)c(C(=O)/C=C/c2ccc(O)cc2)c1O</smiles>

Xanthohumol D (3)

图式 3 天然产物 3 的合成路线

Scheme 3 Synthetic route of natural product 3<smiles>CC(C)=CCc1ccc(O)c(C(=O)C=Cc2ccc(O)c(CC=C(C)C)c2)c1</smiles>

(1) tetraphenylporphyrin $\mathrm{h} v, \mathrm{O}_{2}, \mathrm{DCM}, 5^{\circ} \mathrm{C}$ (2) $\mathrm{Ph}_{3} \mathrm{P}$, r.t., $55 \%$<smiles>C=C(C)C(O)Cc1cc(/C=C/C(=O)c2cc(CC(O)C(=C)C)c(O)cc2O)ccc1O</smiles>

Angusticornin B (4)

图式 4 天然产物 4 的合成路线

Scheme 4 Synthetic route of natural product 4 


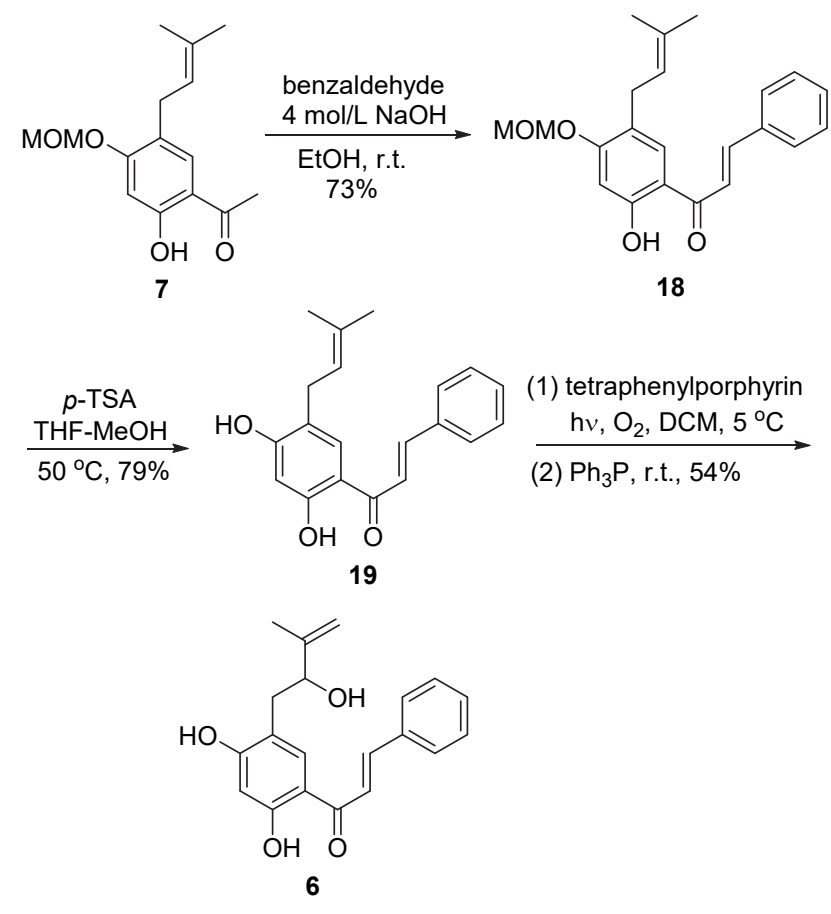

图式 5 化合物 6 的合成路线

Scheme 5 Synthetic route of compound 6

比较), 当查尔酮 $\mathrm{b}$ 环上无取代基时, 抗菌活性再次下降 (衍生物 6). 查尔酮 a 环上有 3 个羟基取代基时, 2 '位甲 氧基取代且 $\mathrm{b}$ 环 4 位羟基取代的活性要明显优于 $4^{\prime}$ 位甲 氧基取代而 $\mathrm{b}$ 环无取代的天然产物(2 和 $\mathbf{3}$ 相比较). 化合 物 3 具有较好的选择性抑制作用, 为该类化合物的构效 关系研究提供了参考.

\section{2 结论}

通过组合化学原理, 以碱性条件下的 ClaisenSchmidt 缩合为关键反应, 首次合成了 4 个羟基异戊烯 基查尔酮类天然产物 $(1 \sim 4)$ 和一个新型衍生物 6 , 其结 构经过 ${ }^{1} \mathrm{H} N M R 、{ }^{13} \mathrm{C} N M R$ 、IR、HRMS 进行了确证. 采 用稀释点样法对所合成的 4 个天然产物 1 4 和 1 个新 型衍生物 6 进行了抗菌活性测试(大肠杆菌 44102、绿脓 杆菌 10104、金黄色葡萄球菌 260003 和枯草芽狍杆菌 63 501). 结果表明: 1、4 和衍生物 6 对革兰氏阳性菌表 现出了一定的抑制活性, 天然产物 3 对枯草芽狍杆菌表 现出了较为明显的选择性抑制作用, 可作为先导化合物 进行抗菌构效关系研究.

\section{3 实验部分}

\section{1 仪器与试剂}

Bruker AVANCE III $400 \mathrm{MHz}$ 核磁共振仪(以 TMS 为内标, 気代氯仿或気代丙酮为溶剂), Varian 7.0T FTMS 高分辨质谱仪, 傅立叶变换红外光谱仪(Nicolet
5700). 本实验所用柱层析硅胶(200 300 目)和薄层板 均为烟台江友化工有限公司生产. 所用药品均购自上海 萨恩化学科技有限公司，试剂均为市售分析纯试剂. 除 特别注明外, 所用溶剂未经无水处理.

\section{2 实验方法}

3.2.1 中间体 1-(2-差基-5-(2-羟基-3-甲基丁基-3-烯1-基)-4-(甲氧甲氧基)苯基)乙烷-1-酮(8)的合成

$5{ }^{\circ} \mathrm{C}$ 下，向化合物 7 (1.0 g, $\left.3.78 \mathrm{mmol}\right)$ 的二氯甲烷 $(210 \mathrm{~mL})$ 溶液中加入四苯基卟啉 $(186 \mathrm{mg}, 0.3 \mathrm{mmol})$. 立 即使用 $500 \mathrm{~W}$ 卤灯照射存有氧气的上述反应体系, 冰水 浴控制温度 $5{ }^{\circ} \mathrm{C}$ 以下摚拌过夜 $(12 \mathrm{~h})$. 加入三苯基膦 $(1.09 \mathrm{~g}, 4.15 \mathrm{mmol})$ 后, 升至室温再搅拌 $3 \mathrm{~h}$. 将反应体 系旋干, 浓缩物通过硅胶柱色谱 $[V$ (乙酸乙酯)：V(石油 醚溶液 $)=1: 9$ ]分离得到 $614 \mathrm{mg}$ 黄色油状物 8 , 产率 58\%. ${ }^{1} \mathrm{H}$ NMR (400 MHz, $\mathrm{CDCl}_{3}$ ) $\delta: 12.57$ (s, $\left.1 \mathrm{H}\right), 7.53$ (s, $1 \mathrm{H}), 6.63(\mathrm{~s}, 1 \mathrm{H}), 5.23(\mathrm{~s}, 2 \mathrm{H}), 4.95(\mathrm{~s}, 1 \mathrm{H}), 4.85(\mathrm{~s}, 1 \mathrm{H})$, $4.28(\mathrm{~s}, 1 \mathrm{H}), 3.48$ (s, 3H), 2.96 (dd, $J=13.9,4.1 \mathrm{~Hz}, 1 \mathrm{H})$, $2.66(\mathrm{dd}, J=13.9,8.6 \mathrm{~Hz}, 1 \mathrm{H}), 2.56(\mathrm{~s}, 3 \mathrm{H}), 1.84$ (d, $J=$ $2.7 \mathrm{~Hz}, 1 \mathrm{H}), 1.82(\mathrm{~s}, 3 \mathrm{H}) ;{ }^{13} \mathrm{C}$ NMR $\left(101 \mathrm{MHz}, \mathrm{CDCl}_{3}\right) \delta$ : $202.95,163.93,161.61,147.25,133.39,118.85,114.26$, 111.01, 102.27, 94.36, 75.25, 56.64, 36.50, 26.51, 18.26; IR (KBr) v: 3538, 3274, 1635, 1559, 1246, $975 \mathrm{~cm}^{-1}$; HRMS (ESI) calcd for $\mathrm{C}_{15} \mathrm{H}_{19} \mathrm{O}_{5}[\mathrm{M}-\mathrm{H}]^{-}$279.1238, found 279.1236 .

3.2.2中间体 $(E$ )-1-(2-差基-5-(2-羟基-3-甲基丁基-3烯-1-基)-4-(甲氧甲氧基)苯基)-3-(4-(甲氧甲氧基)-3(3-甲基丁基-2-烯-1-基)苯基)丙烷-2-烯-1-酮(10)的合 成

$0{ }^{\circ} \mathrm{C}$ 下，向化合物 8 (370 mg, $1.32 \mathrm{mmol}$ )和 9 (371 $\mathrm{mg}, 1.58 \mathrm{mmol})$ 的乙醇 $(12 \mathrm{~mL})$ 溶液中加入 $5 \mathrm{~mol} \cdot \mathrm{L}^{-1}$ $\mathrm{NaOH}(12 \mathrm{~mL}, 60 \mathrm{mmol})$, 缓慢升至室温后搅拌 $36 \mathrm{~h}$. 加 入乙酸乙酯 $(30 \mathrm{~mL})$ 和饱和食盐水 $(20 \mathrm{~mL})$ 进行分液, 水 相用乙酸乙酯 $(50 \mathrm{~mL} \times 2)$ 萃取. 合并有机层, 无水硫酸 钠干燥 $0.5 \mathrm{~h}$. 对有机相进行减压浓缩, 所得粗产品通过 硅胶柱色谱分离 $[V$ (乙酸乙酯 $): V($ 石油醚溶液 $)=1: 9]$ 得到 $249 \mathrm{mg}$ 黄色油状物 10, 产率 38\%. ${ }^{1} \mathrm{H}$ NMR (400 $\left.\mathrm{MHz}, \mathrm{CDCl}_{3}\right) \delta 13.39$ (s, 1H), $7.83(\mathrm{dd}, J=14.9,7.9 \mathrm{~Hz}$, $1 \mathrm{H}), 7.71(\mathrm{~s}, 1 \mathrm{H}), 7.53 \sim 7.39(\mathrm{~m}, 3 \mathrm{H}), 7.16 \sim 7.05(\mathrm{~m}$, $1 \mathrm{H}), 6.71 \sim 6.62(\mathrm{~m}, 1 \mathrm{H}), 5.37 \sim 5.28(\mathrm{~m}, 1 \mathrm{H}), 5.28 \sim 5.16$ (m, 4H), 4.99 (s, 1H), 4.88 (s, 1H), 4.33 (dd, $J=8.3,3.5$ $\mathrm{Hz}, 1 \mathrm{H}), 3.54 \sim 3.43(\mathrm{~m}, 6 \mathrm{H}), 3.38(\mathrm{~d}, J=7.2 \mathrm{~Hz}, 2 \mathrm{H})$, $3.07 \sim 2.98(\mathrm{~m}, 1 \mathrm{H}), 2.70(\mathrm{~m}, 1 \mathrm{H}), 1.85(\mathrm{~s}, 3 \mathrm{H}), 1.74(\mathrm{~s}$, $3 \mathrm{H}), 1.74(\mathrm{~s}, 3 \mathrm{H}) ;{ }^{13} \mathrm{C} \mathrm{NMR}\left(101 \mathrm{MHz}, \mathrm{CDCl}_{3}\right) \delta: 192.21$, $165.35,161.52,157.30,147.29,144.97,133.25,132.24$, $131.52,130.42,128.31,128.14,122.02,118.65,118.09$, 
$114.53,113.89,110.98,102.53,94.38,94.16,75.25,56.66$, 56.29, 36.75, 28.82, 25.94, 18.34, 17.99; IR (KBr) v: 3227, 2930, 1639, 1371, 1267, 1152, $957 \mathrm{~cm}^{-1}$; HRMS (ESI) calcd for $\mathrm{C}_{29} \mathrm{H}_{35} \mathrm{O}_{7}[\mathrm{M}-\mathrm{H}]^{-}$495.2388, found 495.2384.

\subsubsection{Bartericin A (1) 的合成}

向化合物 10 (314 mg, $0.63 \mathrm{mmol})$ 的 THF-MeOH (16 $\mathrm{mL}, V: V=1: 1)$ 溶液中加入对甲苯磺酸 $(218 \mathrm{mg}, 1.26$ $\mathrm{mmol})$, 反应体系在 $50{ }^{\circ} \mathrm{C}$ 下搅拌 $8 \mathrm{~h}$. 加入乙酸乙酯 $(30$ $\mathrm{mL})$ 和饱和食盐水 $(20 \mathrm{~mL})$ 进行分液, 水相用乙酸乙酯 $(50 \mathrm{~mL} \times 2)$ 萃取. 合并有机层, 无水硫酸钠干燥 $0.5 \mathrm{~h}$. 对有机相进行减压浓缩, 所得残余物通过硅胶柱色谱分 离 $[V($ 乙酸乙酯 $): V($ 石油醚溶液 $)=1: 8$ ]得到 $193 \mathrm{mg}$ 黄色油状物 Bartericin A, 产率 75\%. ${ }^{1} \mathrm{H}$ NMR (400 MHz, Acetone- $\left.d_{6}\right) \delta$ : $13.49(\mathrm{~s}, 1 \mathrm{H}), 9.97(\mathrm{~s}, 1 \mathrm{H}), 8.94(\mathrm{~s}, 1 \mathrm{H})$, $8.00(\mathrm{~d}, J=12.3 \mathrm{~Hz}, 1 \mathrm{H}), 7.81$ (d, $J=15.3 \mathrm{~Hz}, 1 \mathrm{H}), 7.74$ $(\mathrm{d}, J=15.3 \mathrm{~Hz}, 1 \mathrm{H}), 7.61 \sim 7.50(\mathrm{~m}, 2 \mathrm{H}), 6.92(\mathrm{~d}, J=8.2$ $\mathrm{Hz}, 1 \mathrm{H}), 6.34(\mathrm{~s}, 1 \mathrm{H}), 5.39 \sim 5.34(\mathrm{~m}, 1 \mathrm{H}), 5.14(\mathrm{~s}, 1 \mathrm{H})$, $4.97(\mathrm{~s}, 1 \mathrm{H}), 4.80(\mathrm{~s}, 1 \mathrm{H}), 4.41(\mathrm{~d}, J=5.1 \mathrm{~Hz}, 1 \mathrm{H}), 3.36$ (d, $J=7.2 \mathrm{~Hz}, 2 \mathrm{H}), 2.95 \sim 2.90(\mathrm{~m}, 1 \mathrm{H}), 2.86 \sim 2.80(\mathrm{~m}, 1 \mathrm{H})$, $1.81(\mathrm{~d}, J=8.3 \mathrm{~Hz}, 3 \mathrm{H}), 1.73(\mathrm{~d}, J=12.6 \mathrm{~Hz}, 6 \mathrm{H}) ;{ }^{13} \mathrm{C}$ NMR (101 MHz, Acetone- $\left.d_{6}\right) \delta: 192.46,165.97,164.19$, $158.37,148.07,145.04,134.01,132.66,131.52,129.36$, $128.73,127.37,122.95,118.78,117.90,116.04,114.08$, $110.49,104.10,76.28,37.81,28.75,25.62,18.03,17.61$; IR (KBr) v: 3343, 1632, 1430, 1256, $1137 \mathrm{~cm}^{-1}$; HRMS (ESI) calcd for $\mathrm{C}_{25} \mathrm{H}_{27} \mathrm{O}_{5}[\mathrm{M}-\mathrm{H}]^{-}$407.1864, found 407.1865 .

3.2.4 中间体 1-(2-着基-4-差基-6-(甲氧甲氧基)-3-(3甲基丁基-2-烯-1-基)苯基)乙烷-1-酮(12)的合成

$20{ }^{\circ} \mathrm{C}$ 下, 向化合物 $11(2.19 \mathrm{~g}, 8.75 \mathrm{mmol})$ 和 $N, N$-二 异丙基乙胺 $(5.7 \mathrm{~mL}, 35.0 \mathrm{mmol})$ 的二氯甲烷 $(80 \mathrm{~mL})$ 溶液 中加入氯甲基甲醚 $(1.33 \mathrm{~mL}, 17.5 \mathrm{mmol})$. 温度升至 $50{ }^{\circ} \mathrm{C}$, 搅拌 $8 \mathrm{~h}$ 后冷却至室温, 向反应体系中缓慢加 入水 $(30 \mathrm{~mL})$. 用二氯甲烷 $(80 \mathrm{~mL} \times 3)$ 萃取, 合并有机 相, 依次使用饱和 $\mathrm{NH}_{4} \mathrm{Cl}$ 溶液 $(30 \mathrm{~mL})$ 和水 $(20 \mathrm{~mL})$ 各洗 涤 1 次, 无水硫酸钠干燥, 减压浓缩的粗产品通过硅胶 柱色谱分离 $[V$ (乙酸乙酯 $): V$ (石油醚溶液 $)=1: 50]$ 得到 $1.54 \mathrm{~g}$ 黄色油状物 12, 产率 60\%. ${ }^{1} \mathrm{H}$ NMR (400 MHz, $\left.\mathrm{CDCl}_{3}\right) \delta: 13.79(\mathrm{~s}, 1 \mathrm{H}), 6.24(\mathrm{~s}, 1 \mathrm{H}), 5.27(\mathrm{~s}, 2 \mathrm{H}), 5.17(\mathrm{t}$, $J=6.8 \mathrm{~Hz}, 1 \mathrm{H}), 3.85$ (d, $J=8.6 \mathrm{~Hz}, 3 \mathrm{H}), 3.51$ (s, 3H), 3.27 (d, J=7.0 Hz, 2H), 2.64 (s, 3H), 1.76 (s, 3H), 1.66 (s, 3H); ${ }^{13} \mathrm{C}$ NMR (101 MHz, $\left.\mathrm{CDCl}_{3}\right) \delta: 203.37,163.27,159.40$, $131.59,122.66,110.76,106.41,94.60,88.93,56.60,55.70$, $33.26,25.93,21.45,17.87$; IR (KBr) v: 2925, 1619, 1420, $1270,1113 \mathrm{~cm}^{-1}$; HRMS (ESI) calcd for $\mathrm{C}_{16} \mathrm{H}_{21} \mathrm{O}_{5}[\mathrm{M}-$
H] ${ }^{-}$293.1394, found 293.1397.

3.2.5中间体 $(E)$-1-(2- 羟基-4-甲氧基-6-(甲氧甲氧 基)-3-(3-甲基丁基-2-烯-1-基)苯基)-3-苯基丙烷-2-烯1 -酮(13) 的合成

$0{ }^{\circ} \mathrm{C}$ 下，向化合物 12 (1.5 g, $\left.5.1 \mathrm{mmol}\right)$ 和苯甲醛 $(0.68 \mathrm{~mL}, 6.62 \mathrm{mmol})$ 的乙醇 $(40 \mathrm{~mL})$ 溶液中加入 $5 \mathrm{~mol}$ • $\mathrm{L}^{-1} \mathrm{NaOH}$ (40 mL, $\left.204 \mathrm{mmol}\right)$, 升至室温后搅拌 $20 \mathrm{~h}$. 加 入乙酸乙酯 $(40 \mathrm{~mL})$ 和饱和食盐水 $(30 \mathrm{~mL})$ 进行分液. 水 相用乙酸乙酯 $(80 \mathrm{~mL} \times 2)$ 萃取. 合并有机相, 无水硫酸 钠干燥 $0.5 \mathrm{~h}$, 对有机相进行浓缩, 所得粗产品通过硅胶 柱色谱分离 $[V$ (乙酸乙酯) $: V$ (石油醚溶液 $)=1: 30]$ 得到 $1.67 \mathrm{~g}$ 黄色油状物 13, 产率 86\%. ${ }^{1} \mathrm{H}$ NMR (400 MHz, $\left.\mathrm{CDCl}_{3}\right) \delta: 13.77(\mathrm{~s}, 1 \mathrm{H}), 7.90(\mathrm{~d}, J=15.6 \mathrm{~Hz}, 1 \mathrm{H}), 7.77$ (d, $J=15.6 \mathrm{~Hz}, 1 \mathrm{H}), 7.66 \sim 7.54(\mathrm{~m}, 2 \mathrm{H}), 7.40(\mathrm{~d}, J=6.5 \mathrm{~Hz}$, $3 \mathrm{H}), 6.25(\mathrm{~s}, 1 \mathrm{H}), 5.30(\mathrm{~s}, 2 \mathrm{H}), 5.22(\mathrm{~s}, 1 \mathrm{H}), 3.88(\mathrm{~s}, 3 \mathrm{H})$, 3.53 (s, 3H), 3.31 (d, J=7.0 Hz, 2H), 1.79 (s, 3H), 1.68 (s, $3 \mathrm{H}) ;{ }^{13} \mathrm{C} \mathrm{NMR}\left(101 \mathrm{MHz}, \mathrm{CDCl}_{3}\right) \delta: 193.27,163.64$, $163.36,158.89,142.03,135.70,131.66,130.15,129.04$, $128.37,127.92,122.66,111.25,107.24,95.44,89.92$, 56.82, 55.75, 25.95, 21.60, 17.91; IR (KBr) v: 1630, 1559, 1333, 1048, $925 \mathrm{~cm}^{-1}$; HRMS (ESI) calcd for $\mathrm{C}_{23} \mathrm{H}_{25} \mathrm{O}_{5}$ $[\mathrm{M}-\mathrm{H}]^{-}$381.1707, found 381.1708 .

3.2.6中间体 $(E)$-1-(2-着基-3-(2-着基-3-甲基丁基-3烯-1-基)-4-甲氧基-6-(甲氧甲氧基)苯基)-3-苯基丙烷2-烯-1-酮(14)的合成

$5{ }^{\circ} \mathrm{C}$ 下，向化合物 $\mathbf{1 3}(1.29 \mathrm{~g}, 3.37 \mathrm{mmol})$ 的二氯甲 烷(350 mL)溶液中加入四苯基卟啉(166 mg, $0.27 \mathrm{mmol}$ ), 立即使用 $500 \mathrm{~W}$ 卤灯照射存有氧气的上述反应体系, 冰 水浴控制温度 $5{ }^{\circ} \mathrm{C}$ 以下搅拌过夜 $(12 \mathrm{~h})$. 加入三苯基膦 (973 mg, $3.7 \mathrm{mmol}$ )后, 升至室温再搅拌 $3 \mathrm{~h}$. 将反应溶 液旋干, 残余物通过硅胶柱色谱 $[V$ (乙酸乙酯) : $V$ (石油 醚溶液 $)=1: 10]$ 分离得到 $738 \mathrm{mg}$ 黄色固体 14, 产率 55\%. m.p. $80 \sim 82{ }^{\circ} \mathrm{C} ;{ }^{1} \mathrm{H}$ NMR (400 MHz, $\left.\mathrm{CDCl}_{3}\right) \delta$ : 14.12 (s, 1H), 7.90 (d, $J=15.6 \mathrm{~Hz}, 1 \mathrm{H}), 7.79$ (d, $J=15.6$ $\mathrm{Hz}, 1 \mathrm{H}), 7.67 \sim 7.48(\mathrm{~m}, 2 \mathrm{H}), 7.47 \sim 7.32(\mathrm{~m}, 3 \mathrm{H}), 6.28(\mathrm{~s}$, $1 \mathrm{H}), 5.32(\mathrm{~s}, 2 \mathrm{H}), 5.00(\mathrm{~s}, 1 \mathrm{H}), 4.82(\mathrm{~s}, 1 \mathrm{H}), 4.24(\mathrm{dd}, J=$ 8.8, 3.2 Hz, 1H), 3.87 (d, $J=8.8 \mathrm{~Hz}, 3 \mathrm{H}), 3.53$ (d, $J=8.9$ $\mathrm{Hz}, 3 \mathrm{H}), 2.97$ (dd, $J=13.8,3.6 \mathrm{~Hz}, 1 \mathrm{H}), 2.86$ (dd, $J=13.9$, $9.0 \mathrm{~Hz}, 1 \mathrm{H}), 1.85(\mathrm{~s}, 3 \mathrm{H}) ;{ }^{13} \mathrm{C} \mathrm{NMR}\left(101 \mathrm{MHz}, \mathrm{CDCl}_{3}\right) \delta$ : $193.35,164.23,163.86,159.42,148.02,142.58,135.57$, $130.31,129.07,128.43,127.61,110.01,108.42,107.08$, 95.38, 89.99, 76.01, 56.88, 55.87, 29.29, 18.25; IR (KBr) v: 2921, 1636, 1563, 1234, 1104, $974 \mathrm{~cm}^{-1}$; HRMS (ESI) calcd for $\mathrm{C}_{23} \mathrm{H}_{25} \mathrm{O}_{6}[\mathrm{M}-\mathrm{H}]^{-}$397.1657, found 397.1655 . 
3.2.7 天然产物 $2^{\prime}, 6^{\prime}$-二羟基-5'-(2"-羟基-3"-甲基-3"丁烯基)-4'-甲氧基查尔酮(2)的合成

向化合物 14 (644 mg, $1.62 \mathrm{mmol}$ )的 THF-MeOH (共 $26 \mathrm{~mL}, V: V=1: 1$ ) 溶液中加入对甲苯磺酸 $(334 \mathrm{mg}$, $1.94 \mathrm{mmol}$ ), 在 $50{ }^{\circ} \mathrm{C}$ 下搅拌 $5 \mathrm{~h}$, 冷却至室温, 加入乙 酸乙酯 $(30 \mathrm{~mL})$ 和饱和食盐水 $(20 \mathrm{~mL})$ 分液, 水相使用乙 酸乙酯 $(50 \mathrm{~mL} \times 2)$ 萃取, 合并有机相, 无水硫酸钠干燥, 对有机相进行浓缩, 所得残余物通过硅胶柱色谱分离 [ $V$ (乙酸乙酯) $: V$ (石油醚溶液 $)=1: 12$ ] 得到 $453 \mathrm{mg}$ 黄 色固体 2, 产率 79\%. m.p. 146 $148{ }^{\circ} \mathrm{C} ;{ }^{1} \mathrm{H}$ NMR (400 $\mathrm{MHz}$, Acetone- $d_{6}$ ) $\delta: 12.84$ (brs, $\left.1 \mathrm{H}\right), 11.65$ (brs, $1 \mathrm{H}$ ), 8.30 (d, $J=15.6 \mathrm{~Hz}, 1 \mathrm{H}), 7.79$ (d, $J=15.7 \mathrm{~Hz}, 1 \mathrm{H}), 7.75 \sim 7.67$ (m, 2H), $7.49 \sim 7.40(\mathrm{~m}, 3 \mathrm{H}), 6.13(\mathrm{~s}, 1 \mathrm{H}), 5.40(\mathrm{brs}, 1 \mathrm{H})$, $4.93(\mathrm{~s}, 1 \mathrm{H}), 4.77(\mathrm{~s}, 1 \mathrm{H}), 4.31(\mathrm{dd}, J=8.3,3.0 \mathrm{~Hz}, 1 \mathrm{H})$, 3.86 (s, 3H), 3.00 (dd, $J=14.4,3.2 \mathrm{~Hz}, 1 \mathrm{H}), 2.93(\mathrm{~s}, 1 \mathrm{H})$, 2.76 (dd, $J=14.4,8.3 \mathrm{~Hz}, 1 \mathrm{H}), 1.81$ (d, $J=8.6 \mathrm{~Hz}, 3 \mathrm{H})$; ${ }^{13} \mathrm{C}$ NMR (101 MHz, Acetone- $\left.d_{6}\right) \delta$ : 193.59, 165.11, $164.84,161.75,148.00,142.40,136.19,130.64,129.52$, $128.88,128.36,110.05,106.60,106.54,92.15,77.02$, 55.93, 29.61, 17.94; IR (KBr) v: 3455, 2925, 1613, 1390, $1055,971 \mathrm{~cm}^{-1}$; HRMS (ESI) calcd for $\mathrm{C}_{21} \mathrm{H}_{21} \mathrm{O}_{5}[\mathrm{M}-$ $\mathrm{H}]^{-}$353.1394, found 353.1396 .

3.2.8中间体 $(E)$-1-(3-(2-差基-3-甲基丁基-3-烯-1基)-6-甲氧基-2,4-二 (甲氧甲氧基)苯基)-3-(4-(甲氧甲 氧基)苯基)丙烷-2-烯-1-酮(16)的合成

$5{ }^{\circ} \mathrm{C}$ 下，向化合物 $\mathbf{1 5}(830 \mathrm{mg}, 1.71 \mathrm{mmol})$ 二氯甲烷 $(180 \mathrm{~mL})$ 溶液中加入四苯基卟啉 $(84 \mathrm{mg}, 0.14 \mathrm{mmol})$, 立 即使用 $500 \mathrm{~W}$ 卤灯照射存有氧气的上述反应体系, 冰水 浴控制温度 $5{ }^{\circ} \mathrm{C}$ 以下搅拌过夜 $(12 \mathrm{~h})$. 加入三苯基膦 (493 mg, $1.88 \mathrm{mmol}$ )后, 升至室温再摚拌 $3 \mathrm{~h}$. 将反应溶 液旋干, 残余物通过硅胶柱色谱 $[V$ (乙酸乙酯) $: V$ (石油 醚溶液 $)=1: 6$ ] 得到 $360 \mathrm{mg}$ 黄色固体 16, 产率 $42 \%$. m.p. $42 \sim 44{ }^{\circ} \mathrm{C} ;{ }^{1} \mathrm{H}$ NMR $\left(400 \mathrm{MHz}, \mathrm{CDCl}_{3}\right) \delta$ : $7.48(\mathrm{~d}$, $J=8.6 \mathrm{~Hz}, 2 \mathrm{H}), 7.33$ (d, $J=16.0 \mathrm{~Hz}, 1 \mathrm{H}), 7.02(\mathrm{~d}, J=8.5$ $\mathrm{Hz}, 2 \mathrm{H}), 6.87(\mathrm{~d}, J=16.0 \mathrm{~Hz}, 1 \mathrm{H}), 6.63(\mathrm{~s}, 1 \mathrm{H}), 5.26(\mathrm{~s}$, $2 \mathrm{H}), 5.19(\mathrm{~s}, 2 \mathrm{H}), 5.02 \sim 4.92(\mathrm{~m}, 3 \mathrm{H}), 4.84(\mathrm{~s}, 1 \mathrm{H}), 4.36 \sim$ $4.28(\mathrm{~m}, 1 \mathrm{H}), 3.77$ (s, 3H), 3.53 (s, 3H), 3.49 (s, 3H), 3.47 (s, 3H), 3.04 2.87 (m, 3H), $1.85(\mathrm{~s}, 3 \mathrm{H}) ;{ }^{13} \mathrm{C}$ NMR $(101$ $\left.\mathrm{MHz}, \mathrm{CDCl}_{3}\right) \delta: 194.30,159.27,157.95,156.99,155.56$, $148.33,144.71,130.26,128.61,127.01,118.08,116.59$, 114.40, 109.99, 101.40, 95.14, 94.90, 94.29, 75.41, 57.64, $56.47,56.29,56.18,30.73,18.40$; IR (KBr) v: 3470, 2939, 1599, 1510, 1239, $1151 \mathrm{~cm}^{-1}$; HRMS (ESI) calcd for $\mathrm{C}_{27} \mathrm{H}_{35} \mathrm{O}_{9}[\mathrm{M}+\mathrm{H}]^{+}$503.2276, found 503.2273.

\subsubsection{Xanthohumol D (3) 的合成}

向化合物 16 (260 mg, $0.52 \mathrm{mmol}$ )的 THF-MeOH (共 $9 \mathrm{~mL}, V: V=1: 1$ )溶液中加入对甲苯磺酸 $(312 \mathrm{mg}, 1.81$ $\mathrm{mmol})$, 反应体系在 $50{ }^{\circ} \mathrm{C}$ 下搅拌 $8 \mathrm{~h}$. 加入乙酸乙酯 $(20$ $\mathrm{mL})$ 和饱和食盐水 $(20 \mathrm{~mL})$ 进行分液. 水相用乙酸乙酯 $(40 \mathrm{~mL} \times 2)$ 萃取. 合并有机层, 无水硫酸钠干燥, 有机 相进行减压浓缩, 所得残余物通过硅胶柱色谱分离 $[V($ 乙酸乙酯 $): V($ 石油醚溶液 $)=1 ： 8$ ] 得到 $168 \mathrm{mg}$ 黄色 固体 Xanthohumol D, 产率 76\%. m.p. $215 \sim 217{ }^{\circ} \mathrm{C} ;{ }^{1} \mathrm{H}$ NMR (400 MHz, Acetone- $d_{6}$ ) $\delta: 14.99$ (s, 1H), 10.04 (s, 1H), 8.96 (s, 1H), 7.91 (d, $J=15.5 \mathrm{~Hz}, 1 \mathrm{H}), 7.76$ (d, $J=$ $15.5 \mathrm{~Hz}, 1 \mathrm{H}), 7.63$ (s, 1H), 7.61 (s, 1H), $6.93(\mathrm{~s}, 1 \mathrm{H}), 6.91$ (s, 1H), $6.08(\mathrm{~s}, 1 \mathrm{H}), 5.51(\mathrm{~s}, 1 \mathrm{H}), 4.96(\mathrm{~s}, 1 \mathrm{H}), 4.77(\mathrm{~s}$, $1 \mathrm{H}), 4.39 \sim 4.31(\mathrm{~m}, 1 \mathrm{H}), 3.96(\mathrm{~s}, 3 \mathrm{H}), 3.04$ (dd, $J=14.6$, $2.9 \mathrm{~Hz}, 1 \mathrm{H}), 2.82(\mathrm{dd}, J=14.6,8.1 \mathrm{~Hz}, 1 \mathrm{H}), 1.82(\mathrm{~s}, 3 \mathrm{H})$; ${ }^{13} \mathrm{C}$ NMR (101 MHz, Acetone- $\left.d_{6}\right) \delta$ : 193.32, 166.73, $165.05,162.58,160.67,148.34,143.34,131.31,128.12$, 125.39, 116.84, 110.28, 106.68, 106.10, 92.73, 77.01, 56.24, 20.70, 18.50; IR (KBr) v: 2923, 1599, 1435, 1199, $1103 \mathrm{~cm}^{-1}$; HRMS (ESI) calcd for $\mathrm{C}_{21} \mathrm{H}_{21} \mathrm{O}_{6}[\mathrm{M}-\mathrm{H}]^{-}$ 369.1344, found 369.1347.

\subsubsection{0 天然产物 Angusticornin B (4)的合成}

向化合物 17 (540 mg, $1.38 \mathrm{mmol})$ 的二氯甲烷 $(100$ $\mathrm{mL}$ )溶液中加入四苯基卟啉 $(110 \mathrm{mg}, 0.18 \mathrm{mmol})$, 立即 使用 $500 \mathrm{~W}$ 卤灯照射存有氧气的上述反应体系, 冰水浴 控制温度 $5{ }^{\circ} \mathrm{C}$ 以下搅拌 $12 \mathrm{~h}$. 加入三苯基膦 $(493 \mathrm{mg}$, $1.88 \mathrm{mmol}$ ), 升至室温再搅拌 $3 \mathrm{~h}$. 将反应溶液旋干, 残 余物通过硅胶柱色谱 $[V$ ( 乙酸乙酯) $: V($ 石油醚溶液 $)=$ $1: 8$ ] 得到 $321 \mathrm{mg}$ 黄色固体 Angusticornin B, 产率 55\%. m.p. $130 \sim 132{ }^{\circ} \mathrm{C} ;{ }^{1} \mathrm{H}$ NMR $\left(400 \mathrm{MHz}\right.$, Acetone- $\left.d_{6}\right) \delta$ : 13.51 (s, 1H), 9.99 (brs, 1H), 9.48 (brs, 1H), 8.01 (s, 1H), $7.80(\mathrm{~s}, 1 \mathrm{H}), 7.79$ (s, 1H), 7.60 (s, 1H) 7.59 (d, $J=8.6 \mathrm{~Hz}$, $1 \mathrm{H}), 6.89(\mathrm{~d}, J=8.9 \mathrm{~Hz}, 1 \mathrm{H}), 6.35(\mathrm{~s}, 1 \mathrm{H}), 5.16(\mathrm{~s}, 2 \mathrm{H})$, 4.97 (s, 2H), 4.79 (s, 2H), 4.47 4.35 (m, 2H), $2.98 \sim 2.81$ $(\mathrm{m}, 4 \mathrm{H}), 1.81(\mathrm{~s}, 3 \mathrm{H}), 1.80(\mathrm{~s}, 3 \mathrm{H}) ;{ }^{13} \mathrm{C}$ NMR $(101 \mathrm{MHz}$, Acetone- $\left.d_{6}\right) \delta$ : 192.78, 166.27, 164.48, 159.81, 148.35, 148.31, 145.24, 134.31, 133.87, 129.74, 127.57, 127.56, $119.09,118.28,117.54,114.37,110.82,104.39,76.67$, 76.57, 38.65, 38.09, 18.29; IR (KBr) v: 1634, 1554, 1367, 1260, 1112, $1017 \mathrm{~cm}^{-1}$; HRMS (ESI) calcd for $\mathrm{C}_{25} \mathrm{H}_{27} \mathrm{O}_{6}$ $[\mathrm{M}-\mathrm{H}]^{-}$423.1813, found 423.1813.

3.2.11中间体 (E)-1-(2-着基-4-(甲氧甲氧基)-5-(3-甲 基丁基-2-烯-1-基)苯基)-3-苯基丙烷-2-烯-1-酮(18)的 合成

$0{ }^{\circ} \mathrm{C}$ 下，向化合物 7 (150 mg, $\left.0.57 \mathrm{mmol}\right)$ 和苯甲醛 
$(78 \mathrm{mg}, 0.74 \mathrm{mmol})$ 的乙醇 $(5 \mathrm{~mL})$ 溶液中加入 $5 \mathrm{~mol} \cdot \mathrm{L}^{-1}$ $\mathrm{NaOH}(5 \mathrm{~mL}, 25 \mathrm{mmol})$, 升至室温摚拌 $20 \mathrm{~h}$. 加入乙酸 乙酯 $(20 \mathrm{~mL})$ 和饱和食盐水 $(5 \mathrm{~mL})$ 进行分液. 水相用乙 酸乙酯 $(20 \mathrm{~mL} \times 2)$ 萃取, 合并有机层, 无水硫酸钠干燥 $0.5 \mathrm{~h}$, 对有机相进行减压浓缩, 所得残余物通过硅胶柱 色谱分离 $[V$ (乙酸乙酯) $: V$ (石油梄溶液 $)=1: 40]$ 得到 $146 \mathrm{mg}$ 黄色油状物 18, 产率 73\%. ${ }^{1} \mathrm{H}$ NMR (400 MHz, $\left.\mathrm{CDCl}_{3}\right) \delta: 13.23(\mathrm{~s}, 1 \mathrm{H}), 7.88(\mathrm{~d}, J=15.5 \mathrm{~Hz}, 1 \mathrm{H}), 7.68 \sim$ $7.62(\mathrm{~m}, 3 \mathrm{H}), 7.58(\mathrm{~d}, J=15.5 \mathrm{~Hz}, 1 \mathrm{H}), 7.48 \sim 7.40(\mathrm{~m}$, $3 \mathrm{H}), 6.66(\mathrm{~s}, 1 \mathrm{H}), 5.29(\mathrm{~s}, 1 \mathrm{H}), 5.26(\mathrm{~s}, 2 \mathrm{H}), 3.48(\mathrm{~s}, 3 \mathrm{H})$, $3.30(\mathrm{~d}, J=7.2 \mathrm{~Hz}, 2 \mathrm{H}), 1.77(\mathrm{~s}, 3 \mathrm{H}), 1.76(\mathrm{~s}, 3 \mathrm{H}) ;{ }^{13} \mathrm{C}$ NMR (101 MHz, $\left.\mathrm{CDCl}_{3}\right) \delta: 192.03,165.01,161.64$, $144.34,134.97,132.99,130.74,130.23,129.12,128.63$, $122.45,122.15,120.57,114.31,102.27,94.06,56.47$, 28.51, 25.92, 18.01; IR (KBr) v: 2927, 1641, 1365, 1243, 1157, 1074, $979 \mathrm{~cm}^{-1}$; HRMS (ESI) calcd for $\mathrm{C}_{22} \mathrm{H}_{23} \mathrm{O}_{4}$ $[\mathrm{M}-\mathrm{H}]^{-}$351.1602, found 351.1603.

3.2.12中间体 $(E)-1$-(2,4-二羟基-5-(3-甲基丁基-2-烯1-基)苯基)-3-苯基丙烷-2-烯-1-酮(19)的合成

向化合物 18 (1.55 g, $4.4 \mathrm{mmol}$ )的 THF-MeOH (20 $\mathrm{mL}, V: V=1: 1)$ 溶液中加入对甲苯磺酸(1.14 g, 6.6 $\mathrm{mmol})$, 反应体系在 $50{ }^{\circ} \mathrm{C}$ 下搅拌 $8 \mathrm{~h}$. 加入乙酸乙酯 $(100 \mathrm{~mL})$ 和饱和食盐水 $(20 \mathrm{~mL})$ 进行分液. 水相用乙酸 乙酯 $(50 \mathrm{~mL} \times 2)$ 萃取, 合并有机层, 无水硫酸钠干燥 0.5 $\mathrm{h}$, 对有机相进行减压浓缩, 所得残余物通过硅胶柱色 谱分离 $[V$ (乙酸乙酯 $): V$ (石油醚溶液 $)=1: 8]$ 得到 1.07 $\mathrm{g}$ 黄色固体 19, 产率 79\%. m.p. 142 $144{ }^{\circ} \mathrm{C} ;{ }^{1} \mathrm{H} \mathrm{NMR}$ $\left(400 \mathrm{MHz}, \mathrm{CDCl}_{3}\right) \delta: 13.23(\mathrm{~s}, 1 \mathrm{H}), 7.87(\mathrm{~d}, J=15.5 \mathrm{~Hz}$, $1 \mathrm{H}), 7.68 \sim 7.63(\mathrm{~m}, 3 \mathrm{H}), 7.57(\mathrm{~d}, J=15.5 \mathrm{~Hz}, 1 \mathrm{H}), 7.47 \sim$ $7.41(\mathrm{~m}, 3 \mathrm{H}), 6.43(\mathrm{~s}, 1 \mathrm{H}), 6.16(\mathrm{~s}, 1 \mathrm{H}), 5.32(\mathrm{~s}, 1 \mathrm{H}), 3.35$ $(\mathrm{d}, J=7.1 \mathrm{~Hz}, 2 \mathrm{H}), 1.80(\mathrm{~s}, 6 \mathrm{H}) ;{ }^{13} \mathrm{C} \mathrm{NMR}(101 \mathrm{MHz}$, $\mathrm{CDCl}_{3}$ ) $\delta: 192.05,165.10,161.97,144.48,135.57,134.95$, $131.35,130.79,129.14,128.66,121.69,120.55,119.11$, 114.38, 104.19, 29.32, 25.96, 18.11; IR (KBr) v: 3317, 1633, 1568, 1362, 1252, $1161 \mathrm{~cm}^{-1}$; HRMS (ESI) calcd for $\mathrm{C}_{20} \mathrm{H}_{19} \mathrm{O}_{3}[\mathrm{M}-\mathrm{H}]^{-}$307.1340, found 307.1342.

$3.2 .1322^{\prime}, 4^{\prime}$-二着基-5'-(2"'-羟基-3"-甲基-3"-丁烯基)查 尔酮(6)的合成

$5{ }^{\circ} \mathrm{C}$ 下, 向化合物 $19(700 \mathrm{~g}, 2.27 \mathrm{mmol})$ 的二氯甲 烷 (120 mL) 溶液中加入四苯基卟啉(140 mg, 0.227 $\mathrm{mmol})$, 立即使用 $500 \mathrm{~W}$ 卤灯照射存有氧气的上述反应 体系, 冰水浴控制温度 $5{ }^{\circ} \mathrm{C}$ 以下搅拌 $12 \mathrm{~h}$, 加入三苯基 膦(655 mg, $2.5 \mathrm{mmol})$, 升至室温再搅拌 $3 \mathrm{~h}$. 将反应溶 液旋干, 残余物通过硅胶柱色谱 $[V$ (乙酸乙酯) : $V$ (石油 醚溶液) $=1 ： 8$ ]得到 $399 \mathrm{mg}$ 黄色固体 6, 产率 54\%. m.p.
$160 \sim 162{ }^{\circ} \mathrm{C} ;{ }^{1} \mathrm{H}$ NMR (400 MHz, Acetone- $\left.d_{6}\right) \delta: 13.33$ (s, 1H), 10.08 (brs, 1H), 8.09 (s, 1H), 7.97 (d, $J=15.5 \mathrm{~Hz}$, $1 \mathrm{H}), 7.90 \sim 7.78(\mathrm{~m}, 3 \mathrm{H}), 7.49 \sim 7.42(\mathrm{~m}, 3 \mathrm{H}), 6.37(\mathrm{~s}$, 1H), 5.14 (brs, 1H), 4.97 (s, 1H), 4.79 (s, 1H), 4.41 (dd, $J=8.2,3.1 \mathrm{~Hz}, 1 \mathrm{H}), 2.94 \sim 2.78(\mathrm{~m}, 2 \mathrm{H}), 1.81(\mathrm{~s}, 3 \mathrm{H}) ;{ }^{13} \mathrm{C}$ NMR (101 MHz, Acetone- $\left.d_{6}\right) \delta: 192.72,166.43,164.95$, $148.35,144.62,136.00,134.65,131.45,129.88,129.64$, $121.87,119.41,114.35,110.85,104.45,76.59,38.10$, 18.34; IR (KBr) v: 3425, 1638, 1566, 1363, 1288, 1141 $\mathrm{cm}^{-1}$; HRMS (ESI) calcd for $\mathrm{C}_{20} \mathrm{H}_{19} \mathrm{O}_{4}[\mathrm{M}-\mathrm{H}]$ 323.1289, found 323.1288.

\section{3 生物活性测定}

实验所用菌株均从中国医学细菌菌种保藏管理中 心获得, 分别为: Bacillus subtilis [CMCC(B)63 501], Staphylococcus aureus [CMCC(B)260003], Escherichia coli [CMCC(B)44102]和 Pseudomonas aeruginosa [CMCC(B) 10104$]$.

具体测试方法参照文献[24], 以氨苦西林作为阳性 对照, 判定指标为最小抑制浓度 $(\mathrm{MIC}, \mu \mathrm{g} / \mathrm{mL})$, 最小抑 制浓度通过三次试验取平均值得出.

辅助材料(Supporting Information) 文中所有目标化 合物和中间体的 ${ }^{1} \mathrm{H}$ NMR 和 ${ }^{13} \mathrm{C} \mathrm{NMR}$ 谱图. 这些材料可 以免费从本刊网站(http://sioc-journal.cn/)上下载.

\section{References}

[1] Zhuang, C.; Zhang, W.; Sheng, C.; Zhang, W.; Xing, C.; Miao, Z. Chem. Rev., 2017, 117, 7762.

[2] (a) Rozmer, Z.; Perjési, P. N. Phytochem. Rev. 2016; 15, 87. (b) Zhou, B.; Xing, C. Med. Chem. 2015, 5, 388.

[3] Zhang, E.; Wang, M.; Xu, S.; Wang, S.; Zhao, D.; Bai, P.; Cui, D.; Hua, Y.; Wang, Y.; Qin, S.; Liu, H. Chin. J. Org. Chem., 2017, 37, 959 (in Chinese).

(张恩，王铭铭，徐帅民，王上，赵娣，白鹏燕，崔得运，化永刚， 王亚娜，秦上尚，刘宏民，有机化学, 2017, 37, 959.)

[4] (a) Sheng, Q.; Zhao, W.; Zeng, M.; Xie, Z.; Xia, Y.; Cui, D. Chin. J. Org. Chem. 2019, 39, 703 (in Chinese).

(盛琦威, 赵婉秋, 曾明, 谢中袍, 夏雅平, 崔冬梅, 有机化学, 2019, 39, 703.)

(b) Gao, Hui.; Zheng, X.; Qi, Y.; Wang, S.; Wan, C.; Rao, G.; Mao, Z. Chin. J. Org. Chem. 2018, 38, 648 (in Chinese).

(高慧, 郑喜, 祁燕, 王斯, 万春平, 饶高雄, 毛泽伟, 有机化学, 2018, 38, 648.)

[5] Yan, Y.; Xu, Q.; Gao, Y.; Liu, H.; Tang, X. Chin. J. Org. Chem. 2018, 38, 1763 (in Chinese). (严映坤, 徐侨, 高扬, 刘辉, 唐孝荣, 有机化学, 2018, 38, 1763.)

[6] Gao, H.; Zheng, X.; Zhu, P.; Wang, S.; Wan, C.; Rao, G.; Mao, Z. Chin. J. Org. Chem. 2018, 38, 684 (in Chinese). (高慧, 郑喜, 朱萍, 王斯, 万春平, 饶高雄, 毛泽伟, 有机化学, 2018, 38, 684.)

[7] Fu, Y.; Sheng, L.; Gao, L.; Li, J.; Sun, L. Chin. J. Org. Chem. 2019 39, 1029 (in Chinese).

(付洋, 盛丽, 高立信, 李佳, 孙良鹏, 有机化学, 2019, 39, 1029.)

[8] Cushnie, T. P. T.; Lamb, A. J. Int. J. Antimicrob. Agents 2011, 38 , 99. 
[9] Avvila, H. P.; Smânia, E. D. F. A.; Delle Monache, F.; Júnior, A. S. Bioorg. Med. Chem. 2008, 16, 9790.

[10] Wang, S.; Dong, G.; Sheng, C. Chem. Rev. 2019, 119, 4180.

[11] Maier, M. E. Org. Biomol. Chem. 2015, 13, 5302.

[12] Shaffer, C. V.; Cai, S.; Peng, J.; Robles, A. J.; Hartley, R. M.; Powell, D. R.; Du, L.; Cichewicz, R. H.; Mooberry, S. L. J. Nat. Prod. 2016, 79, 531 .

[13] Zhai, J.; Fu, L.; Li, Y.; Zhao, R.; Wang, R.; Deng, H.; Liu, H.; Kong, L.; Chen, Z.; Sang, F. Bioorg. Med. Chem. Lett. 2019, 29, 326.

[14] Fang, B.; Xiao, Z.; Qiu, Y.; Shu, S.; Chen, X.; Chen, X.; Zhuang, F.; Zhao, Y.; Liang, G.; Liu, Z. J. Nat. Prod. 2019, 82, 748.

[15] Ngameni, B.; Ngadjui, B. T.; Folefoc, G. N.; Watchueng, J.; Abegaz, B. M. Phytochemistry 2004, 65, 427.

[16] Ren, Z.; Qi, H.; Shi, Y. Planta Med. 2008, 74, 859.
[17] Stevens, J. F.; Taylor, A. W.; Nickerson, G. B.; Ivancic, M.; Henning, J.; Haunold, A.; Deinzer, M. L. Phytochemistry 2000, 53, 759.

[18] Ngadjui, B. T.; Watchueng, J.; Keumedjio, F.; Ngameni, B.; Simo, I. K.; Abegaz, B. M. Phytochemistry 2005, 66, 687.

[19] Dong, X.; Zhou, X.; Jing, H.; Chen, J.; Liu, T.; Yang, B.; He, Q.; Hu, Y. Eur. J. Med. Chem. 2011, 46, 5949.

[20] Maiti, A.; Cuendet, M.; Croy, V. L.; Endringer, D. C.; Pezzuto, J. M.; Cushman, M. J. Med. Chem. 2007, 50, 2799.

[21] Grayfer, T. D.; Grellier, P.; Mouray, E.; Dodd, R. H.; Dubois, J.; Cariou, K. Org. Lett. 2016, 18, 708

[22] Khupse, R. S.; Erhardt, P. W. J. Nat. Prod. 2007, 70, 1507.

[23] Damodar, K.; Kim, J. K.; Jun, J. G. Chin. Chem. Lett. 2016, 27, 698.

[24] Zhuang, W.; Liu, H.; Li, J.; Chen, L.; Wang, G. Front. Microb. 2017, 8, 2573.

(Li, L.; Fan, Y.) 